\title{
Exploring Vitamin B12 Deficiency in Sleeve Gastrectomy from a Histological Study of a Cadaveric Stomach and Ileum
}

\author{
Dwaipayan Muhuri ${ }^{1^{\star}}$, Gyorgy Nagy ${ }^{2}$, Velma Rawlins $^{3}$, Lisa Sandy ${ }^{3}$ and Peter Bellot ${ }^{4}$ \\ ${ }^{1}$ Ross University School of Medicine, NJ 08830, USA \\ ${ }^{2}$ Department of Anatomy, Ross University School of Medicine, NJ 08830, USA \\ ${ }^{3}$ Department of Research, Ross University School of Medicine, NJ 08830, USA
}

${ }^{4}$ Department of Pathology, Ross University School of Medicine, NJ 08830, USA

"Corresponding author: Dwaipayan Muhuri, BSc, MD Student, Ross University School of Medicine, 4B, 485 S Highway 1 South Iselin, NJ 08830, USA, Tel: (302) 781-2167; E-mail: dwaipayanmuhuri@students.rossu.edu

Rec date: 15 June, 2016; Acc date: 19 July, 2016; Pub date: 26 July, 2016

Copyright: (C) 2016 Muhuri D, et al. This is an open-access article distributed under the terms of the Creative Commons Attribution License, which permits unrestricted use, distribution, and reproduction in any medium, provided the original author and source are credited.

\begin{abstract}
Introduction: Vitamin B12 Deficiency is more commonly found amongst patients who have undergone Roux-enY Gastric Bypasses (RYGB) as compared to those post-Sleeve Gastrectomies (SG). The major difference between SG and RYGB is that the latter greatly bypasses the stomach, whereas the former simply reduces the gastric volume.

Purpose: The aim of this paper is to study the stomach and distal ileum, histologically, in a cadaver with SG to explain the higher rate of incidences of B12 deficiency seen in patient's post-RYGB relative to patient's post-SG. Since the stomach is the major variable in the two procedures, we hypothesize that it has the ability to regenerate and increase its surface area to compensate for the loss of its volume in SG patients.
\end{abstract}

Material and Methods: Tissue biopsies and Hematoxylin and Eosin stains were performed from various anatomical locations of the GI tract, specifically, the fundus, the body, and the antrum of the stomach and from the distal ileum of the small intestine of a cadaver with SG and one without (control).

Results: Compared to the control, the SG cadaver's gastric tissue biopsies were significant for chronic gastritis and hypertrophy of the muscularis externa layer. More importantly, parietal cell hyperplasia and deeper mucosal glands were also noted in the SG cadaver supporting the hypothesis.

Conclusion: The compensatory role of an intact stomach, given its ability to regenerate parietal cells and increase its number in the gastric fundus and body, can be better appreciated in a gastric-sparing procedure such as SG versus RYGB in terms of limiting B12 deficiencies.

Keywords: Vitamin B12 Deficiency; Bariatric surgery; Gastrectomy

\section{Introduction}

Of the two broad categories of Bariatric surgery, gastric banding and gastric stapling, the latter is more permanent as it removes or seals off part of the stomach. The 2 most common type of gastric stapling is Roux-en-Y Gastric Bypass (RYGB) and Sleeve Gastrectomy (SG). From 2008 to 2011 , SG procedures jumped roughly $80 \%$ to 94,689 whereas RYGB procedures actually saw a decline by $7 \%$ [1].

Of the chronic complications for SG, nutritional deficiencies are of particular interest, since fewer such incidences are reported post-SG compared to post-RYGB [2]. Transformation of any part of the GI tract could have adverse effects on the body's absorption capability of nutrients including that of Vitamin B12 or Cobalamin. Deficiency in Vitamin B12 can cause pernicious anemia, which can lead to fatigue and memory loss along with palpitations, dizziness, loss of appetite, numbness, vision loss, and depression.
Interestingly, nutritional assessment, either through meta-analysis [3] or through pilot studies that were done worldwide including the United States [4], United Kingdom [5], and Greece [6], suggests a similar finding when it comes to deficiency of Vitamin B12 in patients after their procedures. B12 Deficiency is more commonly found amongst patients who have undergone RYGB as compared to that post-SG [3-6].

Surgically, the major difference between SG and RYGB is that the latter greatly bypasses the physiological functions of the stomach, whereas the former keeps the stomach intact but by vastly reducing its size [2]. Since the stomach is the major variable in the two procedures and plays an important role in B12 absorption as seen in Figure 1a, we hypothesize that the stomach has the ability to regenerate and increase its surface area to compensate for the loss of its volume in SG patients. Since the stomach is largely avoided as a digestive system in patients who have undergone RYGB procedures, compensation for the lack of B12 absorption would most likely be limited (Figures $1 \mathrm{~b}$ and 1c).

The aim of this paper is to study the cadaveric stomach and distal ileum, histologically, in a patient status-post SG to explain the 
Citation: Muhuri D, Nagy G, Rawlins V, Sandy L, Bellot P (2016) Exploring Vitamin B12 Deficiency in Sleeve Gastrectomy from a Histological Study of a Cadaveric Stomach and lleum. J Nutr Disorders Ther 6: 193. doi:10.4172/2161-0509.1000193

Page 2 of 3

difference seen in B12 deficiency between patients post-SG vs. postRYGB (Figure 1d). The study will help answer two questions: does the body have the ability to compensate for a reduced gastric size and if so, then how?

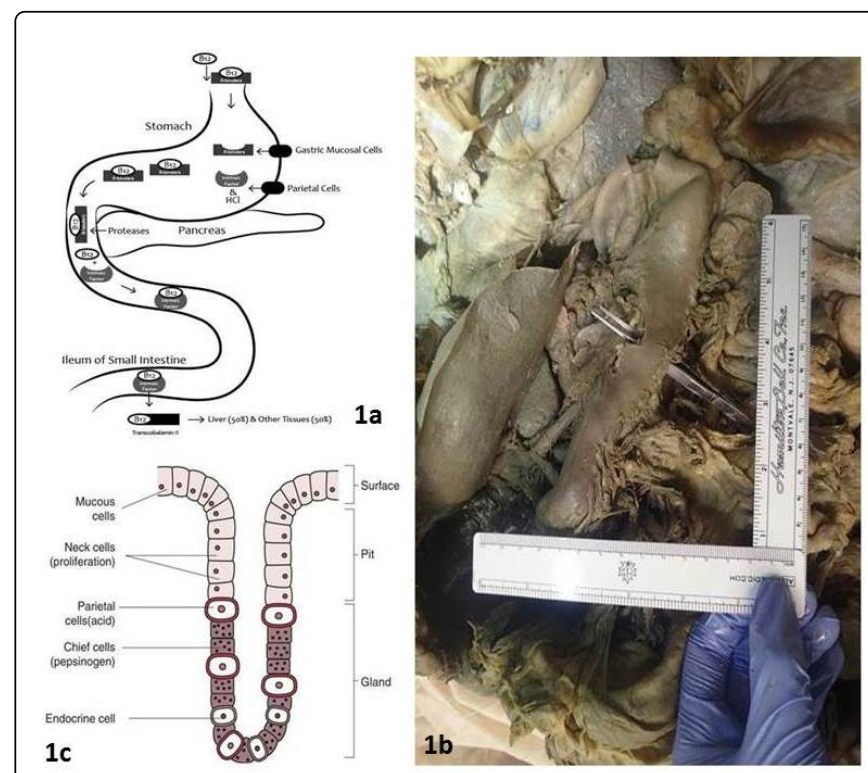

Figure 1: (1a) Normal distribution of the various cell type found in the mucosal pits and glands of gastric fundus and body courtesy of Muir's Textbook of Pathology, 14th Edition 2008; (1b) Photograph of the cadaveric SG stomach used for tissue biopsy; white rings indicate the anatomical location from where tissue was biopsied; (1c)Visual representation of Vitamin B12 absorption pathway courtesy of 2010 Pharmacology Weekly.

\section{Subjects and Methods}

Specimen were collected from two cadavers provided by the Anatomy Department of the Ross University School of Medicine. The cadaver with the SG belonged to a 66-year-old obese male whose cause of death (COD) on record was dementia. The cadaver used as a Control belonged to a 76-year-old obese male whose COD on record was a subdural hematoma. Neither patient had any reported history of Vitamin B12 Deficiency. Past medical history and COD is limited to the information provided above. Tissue biopsies were performed at various anatomical locations as seen in Figure 1c from the GI tract, specifically, the gastric fundus, body (both the greater curvature and the lesser curvature), and antrum and from the distal ileum of the small intestine of both cadavers simultaneously to control for postmortem variables.

Tissue processing and staining protocols set up by the Anatomy Department of Ross University School of Medicine were followed. To prepare the biopsy specimens for a histopathological comparison, they were dehydrated in ascending grades of alcohol and cleared in xylene, before being impregnated with molten paraffin and embedded in blocks. The blocks were left to cool at room temperature prior to being placed in a refrigerator to completely harden. Once solidified, cross sections of the tissues were made and placed on slides. The slides were stained with Hematoxylin and Eosin following proper H\&E staining protocols and then differentiated and dehydrated in ascending alcohol concentrations. Coverslips were placed with mountant before being observed through a light microscope under varying magnifications.

\section{Results}

Histological cross sections of the gastric fundus, body, and antrum indicates chronic gastritis as evidenced by lymphocytes in the mucosal and submucosal layers (Figure 2a). Unlike the Control stomach, a very hypertrophic muscularis externa layer can be appreciated in all areas of the SG stomach under a 40X light microscopic magnification (Figure $2 b)$. The SG stomach biopsies also had deeper pits and glands in the mucosal layer.

With higher magnification at $100 \mathrm{X}$, chief cells (basophilic) and parietal cells (eosinophilic) were clearly identified and differentiated. Hyperplastic parietal cell distribution was noted overall and in particular around the neck (site between the pits and the glands) of the mucosal layers of the fundus and the body of the SG stomach (Figure 2c). The antrum of either the SG or the Control stomach did not have any parietal cell distribution (Figure $2 \mathrm{~d}$ ).

No major histological differences were noted in the distal ileum of either the SG or the Control's small intestine. Lastly, post-mortem tissue alterations including edema and other artifacts were taken into consideration and were deemed to have minimal to no effect on the observed findings.

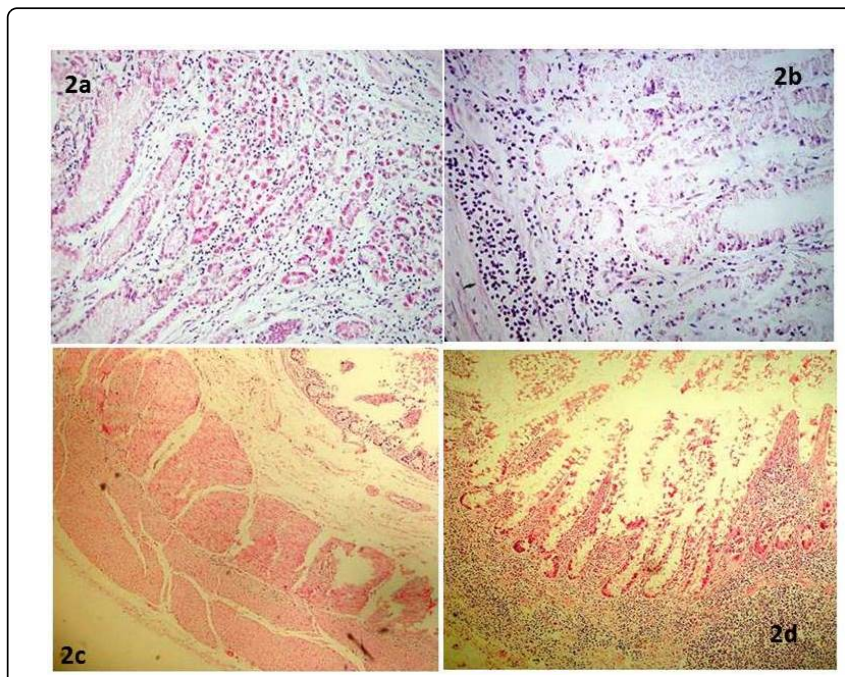

Figure 2: (2a) At 100X magnification, Parietal cell(eosinophilic) hyperplasia were noted in the gastric fundus and body of the sleeve gastrectomy cadaver with $\mathrm{H} \& \mathrm{E}$ stain; (2b) The gastric antrum, however, lacks any parietal cell distribution; (2c) At lower magnification of 40X, a hypertrophic muscularis externa layer; (2d) as well as chronic gastritis; in the mucosal and submucosal layer can be appreciated in the SG cadaver with H\&E stain.

\section{Discussion}

The stomach plays a crucial role in Vitamin B12 absorption. Parietal cells that line the neck of the mucosal glands (Figure 1c) of the gastric fundus and body releases Intrinsic Factor, which binds to B12 in the duodenum and is later recognized by receptors in the distal ileum of the small intestine (Figure 1a). Recognition of the Intrinsic Factors bound to B12 leads to absorption of B12 in the body [7-9]. 
Page 3 of 3

Reduction of gastric volume in an SG procedure means a decrease in the number of parietal cells, which effectively limits the production of Intrinsic Factors required to adequately bind to B12 and avail it for absorption [10-13]. The body, therefore, remains in a compromised state of nutritional deficiency shortly after an SG procedure and requires supplemental therapy to prevent any adverse effects [2,10-13].

However, unlike in RYGB procedures where the entire stomach is bypassed, the stomach in SG procedures is actually utilized, albeit, at a smaller scope [2]. The higher incidence of B12 deficiency in patients after RYGB vs. in patients after SG suggests a unique ability of the stomach to overcome its newly downgraded state after an SG in a very significant way. Parietal cell hyperplasia along with an increased gastric surface area by means of deeper glands seen in a cadaver with SG provides evidence in support of this claim.

In fact, the increased distribution of parietal cells in the neck of the mucosal glands of only the gastric fundus and the gastric body is crucial to understanding the compensatory mechanism through which the human body deals with a decreased gastric volume. Stem cells that can differentiate into surface cells or gland cells are found in the neck of the mucosal glands of the stomach [7,14-16]. Short term B12 deficiency right after a procedure can trigger a response leading to stem cell differentiation into parietal cells, a renewal process that can take weeks $[7,14,15]$. Interestingly, parietal cells were not observed in the antrum of the SG stomach despite the body's desire to respond. In normal histology, Parietal cells are only limited to the gastric fundus and body, whereas, most of the stem cells in the antrum are not programmed for such differentiation [7,14-16].

Although parietal cell hyperplasia as means of gastric compensation provides a compelling argument for the reduced incidences of B12 deficiencies after an SG procedure, alternative theories such as increased secretion of Intrinsic Factor from parietal cells or increased receptor-mediated endocytosis (absorption) of B12 in the distal ileum cannot be ruled out by this study. As such, the complete mechanism explaining gastric compensation for B12 deficiencies is not yet fully grasped and still needs to be studied further.

\section{Limitations and Further Studies}

There were various limitations to this report. Apart from the COD, access to only age and sex were available for either cadaver. Limited medical history of the subjects can blind this study from unforeseen variables, including age when SG was performed or of any prior history of Vitamin B12 Deficiencies. Given the use of cadavers for this study, clinical laboratory tests including blood level measurements for B12 and Methylmalonic Acid to determine B12 Deficiency, a Complete Blood Count to rule out pernicious anemia, and antibody titers against Intrinsic Factors and Parietal Cells for causation were not performed.

This study could be further enhanced with the addition of a postRYGB cadaver as well as by increasing the number of overall cadaveric subjects. Additionally, tissue biopsies from living patients could limit any artefacts from post-mortem tissue alterations, however minimal they might have been in this study. Further studies using immunofluorescence staining to ascertain changes in the distal ileum would be helpful in further establishing the mechanism through which B12 Deficiency is less commonly seen in SG procedures vs. that in RYGB procedures.

\section{Conclusion}

-Histological findings were significant for parietal cell hyperplasia, deeper mucosal glands, muscular hypertrophy, and chronic gastritis in an SG gastric biopsy.

-Parietal cells were noted around the neck of the mucosal glands in only the gastric fundus and body.

-With the intact stomach's ability to not only regenerate parietal cells, but also increase its number in the gastric body of an SG patient, SG is a better alternative to RYGB in terms of limiting Vitamin B12 Deficiency.

\section{References}

1. Lo Menzo E, Szomstein S, Rosenthal RJ (2015) Changing trends in bariatric surgery. Scand J Surg 104: 18-23.

2. Sarkhosh K, Birch DW, Sharma A, Karmali S (2013) Complications associated with laparoscopic sleeve gastrectomy for morbid obesity: a surgeon's guide. Canadian Journal of Surgery 56: 347-352.

3. Kwon Y, Kim H, Lo Menzo E, Park S, Szomstein S, et al. (2014) Anemia, iron and vitamin B12 deficiencies after sleeve gastrectomy compared to Roux-en-Y gastric bypass: a meta-analysis. Surgery for Obesity and Related Diseases 10: 589-597.

4. Saif T, Strain G, Dakin G, Gagner M, Costa R, et al. (2012) Evaluation of nutrient status after laparoscopic sleeve gastrectomy 1, 3, and 5 years after surgery. Surgery for Obesity and Related Diseases 8: 542-547.

5. Eltweri AM, Bowrey DJ, Sutton CD, Graham L, Williams RN (2013) An audit to determine if vitamin b12 supplementation is necessary after sleeve gastrectomy. Springerplus 2: 218 .

6. Alexandrou A, Armeni E, Kouskouni E, Tsoka E, Diamantis T, et al. (2014) Cross-sectional long-term micronutrient deficiencies after sleeve gastrectomy versus Roux-en-Y gastric bypass: A pilot study. Surgery for Obesity and Related Diseases 10: 262-268.

7. Gartner L, Hiatt J (2001) Color Textbook Of Histology. Philadelphia: W.B. Saunders Company, Newyork.

8. Institute of Medicine (1998) Food and Nutrition Board. Dietary Reference Intakes: Thiamin, Riboflavin, Niacin, Vitamin B6, Folate, Vitamin B12, Pantothenic Acid, Biotin, and Choline. Washington, DC: National Academy Press, USA.

9. Klee GG (2000) Cobalamin and folate evaluation: measurement of methylmalonic acid and homocysteine vs vitamin $\mathrm{B}(12)$ and folate. Clin Chem 46: 1277-1283.

10. Carmel R (2008) How I treat cobalamin (vitamin B12) deficiency. Blood 112: 2214-2221.

11. Guéant JL, Safi A, Aimone-Gastin I, Rabesona H, Bronowicki JP, et al. (1997) Autoantibodies in pernicious anemia type I patients recognize sequence 251-256 in human intrinsic factor. Proc Assoc Am Physicians 109: 462-469.

12. Kapadia CR (1995) Vitamin B12 in health and disease: part I--inherited disorders of function, absorption, and transport. Gastroenterologist 3: 329-344.

13. Johnson MA (2007) If high folic acid aggravates vitamin B12 deficiency what should be done about it?. Nutr Rev 65: 451-458.

14. Mills JC, Shivdasani RA (2011) Gastric Epithelial Stem Cells. Gastroenterology 140: 412-424.

15. Hoffmann W (2008) Regeneration of the Gastric Mucosa and its Glands from Stem Cells. Current Medicinal Chemistry 15: 3133-3144.

16. Karam SM (2010) A focus on parietal cells as a renewing cell population. World J Gastroenterol 16: 538-546. 